\title{
La gestion des risques économiques sur le littoral cantabrique au Moyen Âge : un ensemble d'activités ou une multiactivité ?
}

L'exemple des « Quatre Villes de la Côte de la Mer »

Managing economic risk on the Cantabrian coast in the Middle Ages: a group of activities or pluriactivity? The case of Brotherhood of the Four Cities

Jesús Angel Solórzano Telechea et Beatriz Arízaga Bolumburu

\section{(2) OpenEdition}

\section{Édition électronique}

URL : http://journals.openedition.org/abpo/2620

DOI : $10.4000 / a b p o .2620$

ISBN : 978-2-7535-2843-7

ISSN : 2108-6443

Éditeur

Presses universitaires de Rennes

Édition imprimée

Date de publication : 30 juin 2013

Pagination : 97-109

ISBN : 978-2-7535-2838-3

ISSN : 0399-0826

\section{Référence électronique}

Jesús Angel Solórzano Telechea et Beatriz Arízaga Bolumburu, « La gestion des risques économiques sur le littoral cantabrique au Moyen Âge : un ensemble d'activités ou une multiactivité ? », Annales de Bretagne et des Pays de l'Ouest [En ligne], 120-2 | 2013, mis en ligne le 30 juin 2015, consulté le 21 avril 2019. URL : http://journals.openedition.org/abpo/2620 ; DOI : 10.4000/abpo.2620 


\title{
La gestion des risques économiques sur le littoral cantabrique au Moyen Âge : un ensemble d'activités ou une multiactivité?
}

\section{L'exemple des "Quatre Villes de la Côte de la Mer ${ }^{1}$ "}

\author{
Jesús Angel Solórzano Telechea, Beatriz Arízaga Bolumburu \\ Université de Cantabrie - Grupo de Historia Urbana del Área de Historia Medieval
}

Dans l'historiographie espagnole sur les villes portuaires, les visions de l'histoire économique et de la géographie ont longtemps prévalu sur les perspectives sociales, avec des approches centrées sur les fonctions économiques des ports, les produits et les circuits d'échange ${ }^{2}$. Rarement la ville et le port ont été étudiés comme un tout ${ }^{3}$. Il est aujourd'hui nécessaire

1. Traduction littérale de l'expression "Cuatro Villas de la Costa de la Mar » qui englobait les quatre villes portuaires de la province de Cantabrie, à savoir d'est en ouest : Castro Urdiales, Laredo, Santander et San Vincente de la Barquera. Les auteurs remercient Michel Bochaca pour ses commentaires et la relecture d'une version précédente de cet article. La présente étude s'inscrit dans le cadre du projet de recherche Las sociedades urbanas de las ciudades y villas portuarias de la Europa Atlántica en la Baja Edad Media (HAR 2012-31801), dirigé par J. A. Solórzano Telechea et financé par le Ministerio de Economía y Competividad del gobierno de España.

2. Bonachía Hernando, Juan Antonio (coord.), La ciudad medieval. Aspectos de la vida urbana en la Castilla Bajomedieval, Valladolid, Servicio de Publicaciones de la Universidad de Valladolid, 1996. AsENJo GonZÁLEZ, María, « La ciudad medieval castellana. Panorama historiográfico ", Hispania L/2, n 175, 1990, p. 793-808. LóPEZ ALSINA, Fernando, Introducción al fenómeno urbano medieval gallego, a través de tres ejemplos : Mondoñedo, Vivero y Ribadeo, Santiago de Compostela, Universidad de Santiago de Compostela, 1976. ARÍZAGA BolumbURU, Beatriz, El nacimiento de las villas guipuzcoanas en los siglos XIII y XIV : Morfología y funciones urbanas, San Sebastián, Sociedad Guipuzcoana de Ediciones y Publicaciones, 1978. FerReIRA Priegue, Elisa, Galicia en el comercio marítimo medieval, La Coruña, Fundación Barrié de la Maza, 1988. Ruzz De La PeÑa, José Ignacio, Las “ polas " asturianas en la Edad Media. Estudio y Diplomatario, Oviedo, Universidad de Oviedo, 1981. García Oro, José, Galicia en los siglos XIV y XV. Galicia Urbana, II, Pontevedra, Fundación Pedro Barrie de la Maza, 1987.

3. Voir Solórzano Telechea, Jesús Angel, Arízaga Bolumburu, Beatriz (éd.), El fenómeno urbano medieval entre el Cantábrico y el Duero, Santander, AJHC, 2002. ARIZAGA 
de réintroduire les habitants de ces villes dans l'analyse en tant que protagonistes, en utilisant les outils méthodologiques de l'histoire économique, mais aussi de l'histoire sociale et culturelle, ou bien encore de la sociologie et de l'anthropologie. En définitive, il convient d'avoir une vision globale des villes et des sociétés portuaires en termes d'analyse comparée et dans une perspective interdisciplinaire conduite sur la longue durée.

L'objet de la présente étude est de définir la diversité des activités économiques des populations des quatre villes portuaires de Cantabrie : Castro Urdiales, Laredo, Santander et San Vicente de la Barquera ${ }^{4}$, et d'apprécier les formes et les pratiques de la gestion de l'insécurité.

\section{Population et activités urbaines}

Les habitants des villes portuaires de Cantabrie ont, dès les origines de celles-ci, pratiqué simultanément plusieurs types d'activités liées à la mer et la terre. $\mathrm{Au} \mathrm{XI}{ }^{\mathrm{e}}$ siècle, avant même leur fondation par le roi, existaient déjà des emplacements nommés "portus ", c'est-à-dire des lieux d'échange accueillant des navires ${ }^{5}$. Selon la documentation du début de XII ${ }^{\mathrm{e}}$ siècle et les coutumes, il semble que la pêche était une activité complémentaire pour les paysans établis sur la côte ${ }^{6}$. L'Historia Compostelana (vers 1115) qualifie les habitants de la côte de paysans. Malgré la peur des raids sarrasins, il s'agit, comme l'atteste l'extrait suivant, d'un choix délibéré des populations de vivre sur la côte pendant certaines périodes de l'année : "Les paysans du littoral de l'océan abandonnaient la côte depuis la moitié du printemps jusqu'à la moitié de l'automne ou se cachaient dans des grottes avec toute leur famille ${ }^{7}$. " Une telle organisation met en lumière une double activité.

Bolumburu, Beatriz, BochacA, Michel, « Caractères généraux des villes portuaires du nord de la Péninsule Ibérique ", dans Ports Maritimes et ports fluviaux au Moyen Âge, Paris, Presses universitaires de la Sorbonne, 2005, p. 63-78. SolóRZANo TelECHEA, Jesús Angel, "Medieval Seaports of the Atlantic Coast of Spain ", International Journal of Maritime History, XXI, 2009, p. 81-100.

4. Les "Cuatro Villas de la Costa de la Mar" étaient une structure administrative, née à la fin du Moyen Âge et administrée par le corregidor ou lieutenant du Roi.

5. "Tribuo quoque clementer ut habeatis licentiam de pescare per omnes meos pelagos vel defesas, sive per marinos portos, scilicet, in Sancta Maria de Porto, et in por $S$. Emetherii et in illo de S. Martini et de Apleca " (1068). SERRANo, Luciano, El obispado de Burgos y Castilla primitiva desde el siglo $\mathrm{V}$ al XIII, Madrid, Instituto de Valencia de Don Juan, 1935, vol. III, p. 31-33. QuAGHEBEUR, Joëlle, "Le monde littoral breton des $\mathrm{IX}^{\mathrm{e}}$-XI ${ }^{\mathrm{e}}$ siècles ", dans CÉRINo, Christophe, GeISTDOERFER, Aliette, LE BouËDEc, Gérard, Ploux, François (dir.), Entre terre et mer. Sociétés littorales et pluractivités $\left(X v^{e}-X X^{e}\right.$ siècle), Rennes, Presses universitaires de Rennes, 2004, p. 63.

6. SolóRzAno TelecheA, Jesús Angel, "La fundación y promoción de las "villas nuevas" en litoral Atlántico del Norte peninsular durante el reinado de Alfonso X ", dans El mundo urbano en la Castilla del siglo XIII. 750 Aniversario de la Fundación de Ciudad Real. Actas del Congreso, Ayuntamiento de Ciudad Real / Universidad de Sevilla, Ciudad Real, 2006, p. 315-328.

7. Historia Compostelana, Madrid, éd. Emma Falque, 1994. 
Les activités comme la pêche, le commerce, l'agriculture et l'élevage apparaissent dans les coutumes octroyées à Santander, Laredo, Castro Urdiales et San Vicente de la Barquera par le roi Alphonse VIII entre 1163 et $1210^{8}$. La coutume de Laredo autorise les voisins de la ville (vecinos) ${ }^{9}$ à faire circuler librement leur bétail dans tout le royaume. Celle de Santander leur octroie un espace de 15 kilomètres autour de la ville pour défricher, travailler et cultiver des terres et des vignobles ${ }^{10}$. La division du travail était sans doute très faible à l'origine, car peu de métiers sont mentionnés. Une lente spécialisation des activités s'opère au XIII ${ }^{\mathrm{e}}$ siècle ${ }^{11}$. On voit apparaître des mentions de pêcheurs, de marchands, de charpentiers, de tonneliers, lesquels exerçaient parfois une autre activité, terrestre ou maritime ${ }^{12}$. Le secteur économique le plus spécialisé fut sans doute celui de la pêche, avec pour conséquence la naissance des confréries de pêcheurs et de marins au $\mathrm{XIV}^{\mathrm{e}}$ siècle ${ }^{13}$ : confrérie de Saint-Martin à Laredo en 1306, confrérie de SaintVincent à San Vincente de la Barquera en 1330, de Saint-Martin à Santander en 1384 et de Saint-André à Castro Urdiales en $1395^{14}$.

Dès lors, étudier les sociétés urbaines du littoral cantabrique revient à s'intéresser à la complémentarité existant entre la profession déclarée des citadins et leurs autres activités économiques, caractéristique que les

8. Ferreira Priegue, Elisa, "L'incorporation du Nord-Ouest ibérique à la vie économique du golfe de Gascogne ", dans L'aventure maritime, du golfe de Gascogne à Terre-Neuve, Paris, CTHS, 1995, p. 331-340. RUIZ DE LA PEÑA, José Ignacio, "El nacimiento de las villas costeras cántabro-atlánticas y su instrumentación jurídica (siglos XII-XIII) ", dans El Fuero de Laredo en el VIII centenario de su concesión, Santander, Universidad de Cantabria, 2001, p. 156.

9. Le statut juridique du voisin s'apparente à celui du bourgeois.

10. «Per omnes partes regni mei libera ganati vestri habeant pascua tamquam mei propii ganati » (Coutume de Laredo de l'année 1200). Publié par MARTínEz DíEz, Gonzalo, "Fueros locales de la Provincia de Santander ", dans Anuario de Historia del Derecho Español, XLVI, p. 527-608.

11. RuIZ DE LA PEÑA SOLAR, José Ignacio, « El desarrollo urbano y mercantil de las villas cántabras en los siglos XII y XIII ", dans El fuero de Santander y su época. Actas del congreso conmemorativo de su VIII centenario, Santander, Estudio, 1989, p. 257-259. Id., " Nacimiento de las villas de la costa cántabro-atlántica ", Litoral Atlántico, nº 3, 2001, p. 12-21.

12. "La vigne de don Helias, chaudronnier" (en 1274), " charpentier que tu plantes une vigne " (en 1326). FERnÁNDEZ GONZÁLEZ, Lorena, Archivo de la catedral de Santander, Santander, Fundación Marcelino Botín, 1994.

13. Epstein, S. R., "Craft Guilds, Apprenticeship and Technological change in Preindustrial Europe ", The journal of Economic History, 58, 3, 1998, p. 684-713. KIESER, Alfred, "Organizacional, institucional and societal evolution: Medieval Craft Guilds and the Genesis of formal Organizations ", Administrative Science Quarterly, 34, 4, 1989, p. 540564. TRIO, Paul, «Les confréries comme expression de solidarité et de conscience urbaine aux Pays-Bas à la fin du Moyen Âge ", dans Memoria, communitas, civitas. Mémoire et conscience urbaines en Occident à la fin du Moyen Âge, Paris, 2003.

14. Archivo de la Real Audiencia y Chancillería de Valladolid. Sección Pleitos civiles. Fernando Alonso (F), c. 992-1. Fernández GonzÁlez, Lorena, Archivo de la catedral de Santander. (ss. XII-XVI), Santander, Fundación Marcelino Botín, 1998, p. 167-168. CUÑAT CISCAR, Virginia, Documentación medieval de la villa de Laredo. 1200-1500, Santander, Fundación Marcelino Botín, 1998, p. 83-84. 
élites urbaines partageaient avec les gens du commun ${ }^{15}$. Quelles que soient leur profession et leur position sociale, les voisins exerçaient plusieurs activités ${ }^{16}$. En effet, rares sont ceux qui pratiquaient une seule et unique activité professionnelle.

Cependant, les groupes sociaux - oligarchie et commun - adoptaient des stratégies professionnelles différenciées. Composant $90 \%$ de la population des villes portuaires, le commun formait le secteur productif de base de l'économie urbaine ${ }^{17}$. De composition variée, il se caractérise par l'absence de privilèges fiscaux et de participation dans les institutions politiques des villes ${ }^{18}$. Il faut distinguer en son sein les voisins exerçant des activités artisanales et mercantiles (appelés omes ruanos) et dont la position économique était suffisamment assurée pour pouvoir investir dans l'achat de terres ou participer aux frais d'entreprises de pêche, et les simples pêcheurs, les petits artisans (charpentiers, maçons...), les paysans et autres ouvriers et journaliers (omes baxos). Formant le groupe urbain le plus pauvre, ces derniers manquaient de moyens économiques et partageaient de ce fait souvent les revenus de la pêche avec la location et l'exploitation de vergers, de terres et de vignes pour assurer leur subsistance quand ils n'étaient pas en mer. Le cas des élites urbaines (principales) est quelque peu différent puisqu'elles sont en mesure de développer des stratégies complexes de diversification de leurs ressources économiques : commerce, propriété de biens ruraux, prises de participation aux revenus royaux et municipaux, piraterie, guerre, auxquelles s'ajoutait le contrôle des institutions locales qui leur permettait d'intervenir directement dans les affaires des villes, le plus souvent à leur profit.

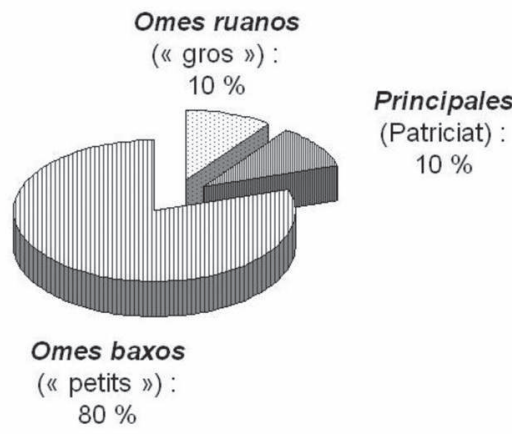
Figure 1 - La structure sociale à Santander selon l'enquête de 1504

15. Arízaga Bolumburu, Beatriz, «El abastecimiento de las villas vizcaínas medievales: política comercial de las villas respecto al entorno y su interior ", dans La ciudad hispánica, I, Madrid, Universidad Complutense de Madrid, 1985, p. 293-316.

16. Solórzano TelecheA, Jesús Ángel, Santander en la Edad Media : patrimonio, parentesco y poder, Santander, Servicio de Publicaciones de la Universidad de Cantabria, 2002.

17. Solórzano Telechea, Jesús Ángel, ARízaga Bolumburu, Beatriz (éd.), "El fenómeno urbano... ", op. cit., p. 241-307.

18. Archivo de la Real Chancillería de Valladolid, Reales Ejecutorias, c. $286 / 32, \mathrm{f}^{\circ} 3 \mathrm{v}^{\circ}-4 \mathrm{v}^{\circ}$. 


\section{Les activités du commun par rapport à la terre}

La majorité des propriétaires de terres situées autour des villes habitait à l'intérieur de l'enceinte urbaine et jouissait du statut de "voisin ". Les propriétaires habitant dans la banlieue (término municipal) étaient très peu nombreux. Plusieurs questions se posent dès lors : à partir de quand et pour quelles raisons les gens du commun se sont-ils intéressés aux propriétés rurales périurbaines? Quels groupes au sein du commun étaient plus particulièrement concernés?

Dans le cas de Santander, il faut attendre le troisième quart du XIV ${ }^{\mathrm{e}}$ siècle pour trouver trace d'achats systématiques de propriétés rurales par les voisins. Jusqu'alors, la transmission des terres s'était faite par des legs testamentaires à l'intérieur du cercle familial. Avant 1360, 85 \% des terres s'échangeaient entre les membres d'une même famille, alors que dans les décennies qui suivent, $63 \%$ changent de mains par achat. Les transactions agraires entre voisins s'imposèrent, donnant naissance à un marché de la terre, dont le point d'expansion maximal à Santander se situe dans le deuxième quart du XV $\mathrm{Xv}^{\mathrm{e}}$ siècle $(70 \% \text { des transactions })^{19}$. Au milieu du XV siècle, le marché de la terre s'est converti pour quelques voisins en un moyen d'accumulation de biens fonciers.

Figure 2 - Achats et locations de terres à Santander de 1350 à 1510

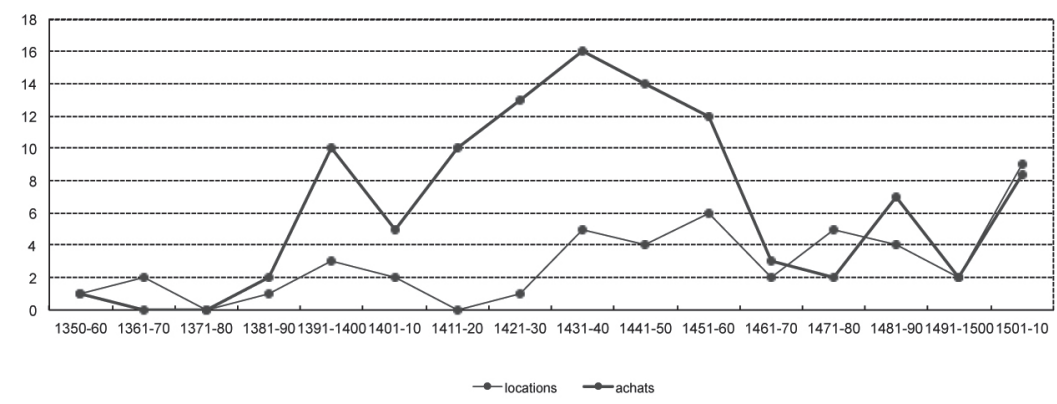

On peut s'interroger sur les raisons économiques de ces investissements. Ils répondent sans aucun doute à un souci de profit, mais également à une recherche de garantie et de sécurité pour les placements opérés. Pendant les années de crise, la production agricole locale a été la source fondamentale d'approvisionnement urbain et le moyen d'enrichissement et d'affermissement d'un petit groupe de voisins. Les achats de terres constituaient enfin un élément de prestige social, renvoyant à un idéal de vie qui permettait d'échapper aux oscillations du marché, voire d'imiter la

19. Solórzano Telechea, Jesús Angel, Santander en la Edad Media..., op. cit., p. 20-31. 
noblesse ${ }^{20}$. C'était le rêve des omes ruanos, groupe qui, de fait, a le mieux surmonté les crises.

De cette façon, nous pouvons établir un lien direct entre la crise économique et l'acquisition de propriétés rurales, en y voyant un moyen de faire face aux malheurs du temps grâce notamment à une diversification des activités. Entre le milieu du XIV ${ }^{\mathrm{e}}$ siècle et le premier quart du XV $\mathrm{XV}^{\mathrm{e}}$ siècle, la part des voisins propriétaires de biens ruraux autour de Santander s'élève jusqu'à atteindre 75 \%. En 1450, une fois les crises passées, leur proportion tombe à $55 \%$. Ce pourcentage repart à la hausse à la fin du XV siècle, quand survient une autre crise économique et démographique causée par une épidémie de peste. Nous pouvons donc constater que le capital urbain tend à investir les campagnes environnantes pendant les années de crise, pour s'en extraire une fois les difficultés passées. Ces investissements étaient l'unique façon de sécuriser sur le long terme des profits économiques somme toute limités.

\section{Figure 3 - Les moyens d'accès à la terre à Santander} aux $X I V^{e}$ et $X V^{e}$ siècles

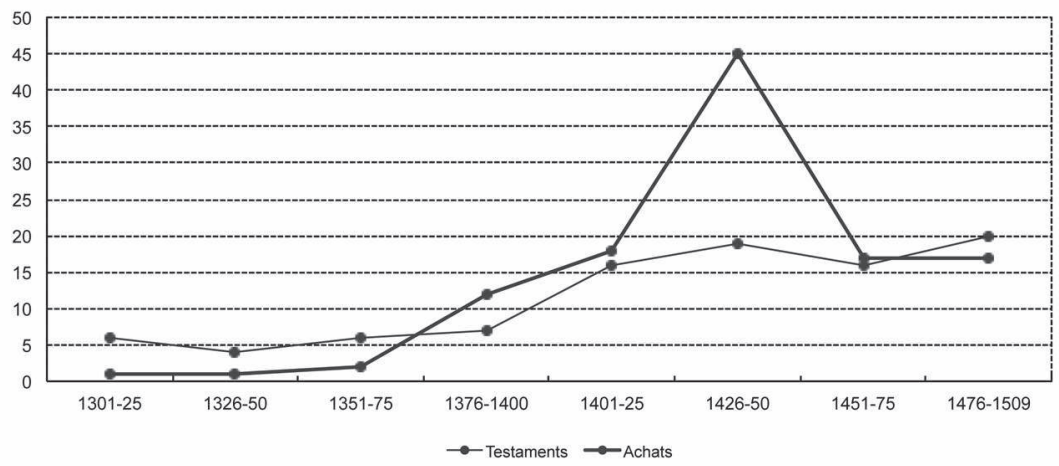

L'achat ou la location de vignes était le fait de voisins de différentes conditions socio-professionnelles, principalement des artisans (tailleurs d'habits, tanneurs, tisserands, forgerons, tonneliers, charpentiers, calfats, tailleurs de pierre, chaudronniers, cordonniers, etc.), mais aussi des notaires, apothicaires, chirurgiens, médecins, avocats, auxquels s'ajoutaient des marchands et des marins. Tous appartenaient au groupe des omes ruanos. Il s'agissait dans la plupart des cas d'exploitations dispersées et de faible superficie. La culture dominante dans la banlieue était la vigne, même si l'on trouve également des vergers et des plantations maraîchères.

20. Asenjo González, María, "Ciudad y territorio en la Castilla bajomedieval. Dinámica socioeconómica ", dans El poder a l'Edat mitjana, Barcelona, Pagés, 2004, p. 173-208. 
Ces biens rapportaient un complément financier non négligeable pour cette frange de la population, essentiellement dédiée à l'artisanat. Il s'agissait d'une forme d'investissement du capital qui leur permettait de participer à la vie économique locale et d'en tirer profit. Le vin rapportait des revenus réguliers, puisqu'il pouvait être facilement vendu. Des privilèges royaux permettaient de l'exporter dans le reste du royaume de Castille et des ordonnances royales et municipales protégeaient les marchands de la concurrence des vins étrangers importés ${ }^{21}$. Outre la possibilité d'écouler leur production sur le marché urbain, l'acquisition de biens ruraux constituait le plus sûr moyen de protéger le capital des fréquentes fluctuations monétaires.

On notera toutefois qu'il n'y avait pas de pêcheurs parmi les petits propriétaires terriens, bien que nous les trouvions occasionnellement comme locataires de vignes ou de vergers. La location était pour eux le moyen d'accéder aux fruits de la terre à défaut de pouvoir acquérir la propriété du sol. Ils pratiquaient l'agriculture, essentiellement la viticulture, comme une activité annexe - ils n'y consacraient qu'un temps limité - pour les besoins de leur propre consommation. Ces activités tant maritimes qu'agricoles avaient un caractère saisonnier qui rythmait la vie de ces sociétés littorales. En général, les pêcheurs travaillaient les champs quand ils ne pouvaient pas sortir en mer. Dans les ordonnances de la ville biscayenne voisine de Bermeo, la municipalité tente d'ailleurs en 1353 d'obliger les pêcheurs à travailler leurs terres les jours où ils ne sortaient pas pêcher ${ }^{22}$. L'arboriculture, notamment la culture des citronniers, constituait également une activité complémentaire.

L'enquête sur la situation de Santander lors de la peste de 1504 montre que les voisins rangeaient les pêcheurs et les paysans parmi les omes baxos. Ces derniers travaillaient de façon saisonnière les vignes, en qualité de journaliers. Une ordonnance municipale de Santander, du début du XvI ${ }^{\mathrm{e}}$ siècle, mentionne la présence de " charpentiers, maçons, ouvriers, journaliers et les autres hommes et femmes et les ouvriers, que l'on a l'habitude à louer

21. ArízAga Bolumburu, Beatriz, " La villa de Laredo : las rentas de sus términos jurisdiccionales en la Edad Media ", dans Os Reinos Ibericos na Idade Media, Porto, Éditeur, 2003, p. 297-304.

22. Id., "La pesca en el País Vasco en la Edad Media ", Itsas Memoria, n ${ }^{\circ}$ 3, 2000, p. 17. Jaime de Labayru, Estanislao, Historia General del Señorío de Bizcaya, Bilbao, La Propaganda, 1897, t. II, p. 820-840. GARCía FERNÁNDEZ, Ernesto, "Las cofradías de oficios en el País Vasco durante la Edad Media (1350-1550) ", Studia Historica. Historia Medieval, 1997, n 15, p. 11-40. TenA GARCíA, María Soledad, "Composición social y articulación interna de las cofradías de pescadores y mareantes. (Un análisis de la explotación de los recursos marítimos en la Marina de Castilla durante la Baja Edad Media) ", Espacio, tiempo y forma. Serie III, Historia medieval, 1995, n 8, p. 114. GARCía FERNÁNDEZ, Ernesto, "Las cofradías de mercaderes, mareantes y pescadores vascas en la Edad Media ", dans Arízaga Bolumburu, Beatriz, Solórzano Telechea, Jesús Angel, Ciudades y villas portuarias del Atlántico en la Edad Media. Nájera. I Encuentros Internacionales del Medievo, Logroño, IER, 2005, p. 257-294. 
pour le travail des champs ${ }^{23}$ ". Deux groupes sont nettement distingués : ceux qui avaient un métier mais qui travaillaient occasionnellement la terre (charpentier, maçons), et ceux qui étaient seulement ouvriers ou journaliers agricoles. On remarquera d'ailleurs qu'il n'y avait pas de pêcheurs parmi eux. De la même façon, les journaliers étaient employés pour l'entretien des infrastructures urbaines. En 1504, le manque de main-d'œuvre, causé par la peste, révèle combien le travail des journaliers était essentiel pour les voisins, ces derniers n'étant pas en mesure d'assurer eux-mêmes l'entretien de toutes leurs terres. La pénurie était tout aussi grave pour la mise en valeur des vignes et des propriétés rurales des élites urbaines et des omes ruanos (l'élite du commun) ${ }^{24}$.

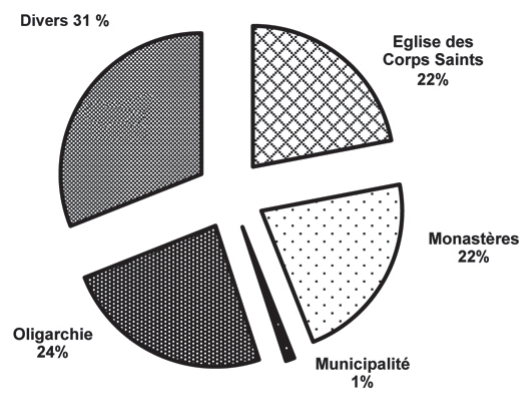

Figure 4 - Les propriétaires des terres dans la banlieue de Santander à la fin du Moyen Âge

\section{Figure 5 - Développement de la propriété rurale dans la banlieue de Santander}

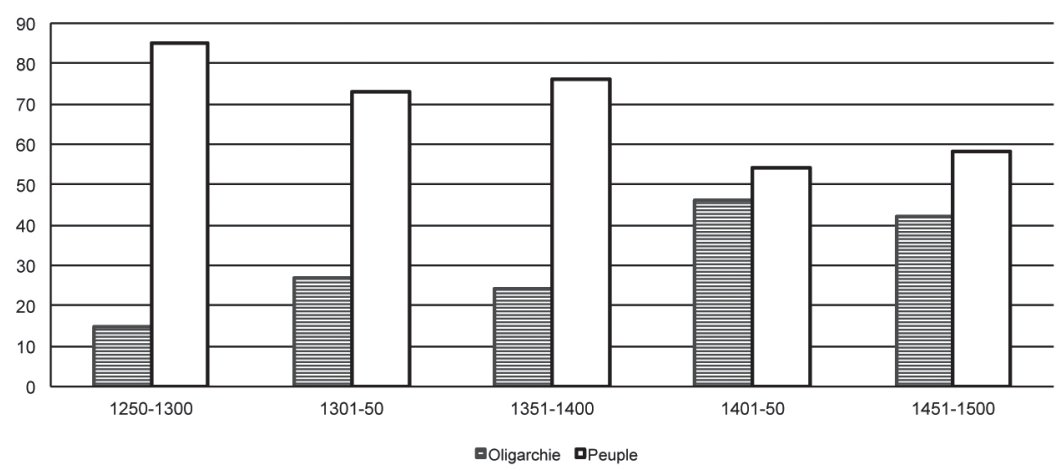

23. Solórzano Telechea, Jesús Angel, Colección diplomática del Archivo Municipal de Santander. Documentación medieval, Santander, Fundación Marcelino Botín, 1995, doc. 255.

24. Ibidem. 
À la fin du Moyen Âge, les terres qui entourent les quatre villes portuaires du littoral cantabrique étaient surtout travaillées par des journaliers, ce qui témoigne d'une structure socioprofessionnelle plus spécialisée qu'auparavant. Un autre exemple de cette évolution nous est fourni au début du $\mathrm{XVI}^{\mathrm{e}}$ siècle. En effet, une décision du conseil municipal de Santander met en lumière le fait que l'embarquement des laines sur le port nuisait au travail des champs, de telle sorte que bon nombre de journaliers avaient délaissé les travaux agricoles préférant être employés comme manutentionnaires. Les pertes étaient évaluées à 2000 ducats ${ }^{25}$. Les travailleurs les plus pauvres pratiquaient une pluriactivité réelle dans les quatre villes de la côte. Ils subvenaient à leurs besoins grâce à l'exercice de plusieurs métiers.

La municipalité a réglementé les conditions de travail - souvent très difficiles - des journaliers au moyen d'ordonnances. Hommes et femmes devaient travailler du lever au coucher du soleil et apporter leurs propres outils, ainsi qu'un repas ${ }^{26}$. Leur situation s'est dégradée pendant le dernier quart du $\mathrm{XV}^{\mathrm{e}}$ siècle avec l'instauration d'une surveillance exercée par un gardien institué par le conseil municipal.

\section{Les activités du commun en relation avec la mer}

Si les historiens ont particulièrement insisté sur la fonction commerciale et mercantile des villes portuaires, il n'en reste pas moins que la pêche demeure la principale activité économique. L'essentiel de la population vivait de la pêche et appartenait au groupe social des omes baxos ; ils se nommaient d'ailleurs " hommes de la mer et travailleurs ${ }^{27}$ ". Environ $80 \%$ des hommes habitant Orio au milieu du $\mathrm{XVI}^{\mathrm{e}}$ siècle étaient des pêcheurs ${ }^{28}$. À Laredo, selon un privilège royal accordé en 1406 à la confrérie des pêcheurs, " il n'y avait pas d'autres métiers dans la ville que ceux de naviguer et pêcher ${ }^{29}$ ". Selon l'enquête sur l'état de la ville de Santander, dressé en 1504, le nombre de pêcheurs s'élevait à 800 voisins, soit $80 \%$ du nombre total de voisins, estimé alors à 1000 selon la même enquête ${ }^{30}$. À Castro Urdiales, d'après l'enquête de 1558 concernant l'impôt royal prélevé sur les ventes de marchandises (alcabalas), la population masculine était composée à $70 \%$ de pêcheurs et de marins, à $11 \%$ de journaliers, $8 \%$ d'artisans et $2 \%$ de paysans ${ }^{31}$.

25. Archives privées de la famille González-Camino (AFGC).

26. Archives Historiques de la Province de Cantabrie (AHPC), Diversos, leg. 52, n ${ }^{\circ} 40$.

27. Solórzano Telechea, Jesús Angel, Vázquez Álvarez, Roberto, Arízaga Bolumburu, Beatriz, San Vicente de la Barquera: una villa en conflicto, Santander, AJHC, 2004, doc. 28.

28. ARÍzAgA Bolumburu, Beatriz, « Las sociedades urbanas en la España medieval », dans XXIX Semana de Estudios Medievales de Estella. Pamplona, Gobierno de Navarra, 2003, p. 219.

29. Cuñat Ciscar, Virginia, Documentación medieval de la villa de Laredo. 1200-1500. Santander, Fundación Marcelino Botín, 1998.

30. SolóRzAno TelecheA, Jesús Angel, Colección diplomática..., op. cit., doc. 255.

31. Baró Pazos, Juan, Galván Rivero, Carmen, Libro de ordenanzas de Castro Urdiales (1519-1572), Santander, Universidad de Cantabria, 2006, p. 53. 
Figure 6 - Population masculine active à Castro Urdiales (1558)

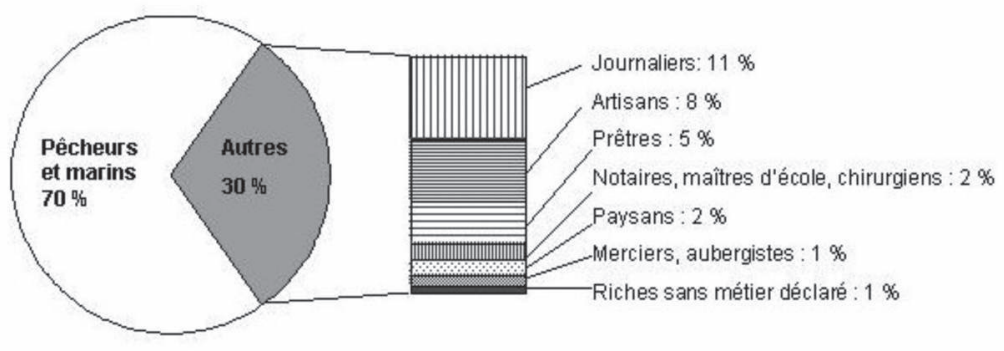

Dans les Quatre Villes de la Mer, les données fournies par les sources fiscales (alcabalas) montrent qu'au moins 50 \% des recettes provenaient de la taxation de la pêche : 54 \% à San Vicente de la Barquera, 53 \% à Castro Urdiales et à Laredo, 48 \% à Santander. Le reste était partagé entre différents produits, parmi lesquels le vin, avec $15 \%$ des recettes, arrivait loin derrière les produits de la pêche ${ }^{32}$. Ces ordres de grandeur montrent que le principal moyen de subsistance des habitants était lié à la pêche et à la vente du poisson, de sorte que les autres activités n'avaient qu'un poids limité dans l'économie des villes. En 1409, un privilège du roi de Castille, Jean II, nous informe d'une plainte déposée par la confrérie des pêcheurs et marins de Saint-Martin de Laredo. Cette dernière le supplie de protéger les confrères d'éventuelles saisies de leurs propriétés, saisies orchestrées en raison des dettes que le conseil municipal de Laredo avait contractées envers le roi et d'autres conseils. Pour cette raison, aucun voisin n'osait aller pêcher et naviguer jusqu'en Galice ou vendre du poisson à Burgos ou toute autre marchandise ailleurs dans le royaume. La confrérie informait le roi que la ville de Laredo risquait de se dépeupler, puisque « il n'y avait que les métiers de la pêche et si le roi ne leur donnait pas sa protection, ils seraient obligés de vivre dans d'autres ports ${ }^{33}$ ".

Le poisson n'était pas seulement un produit alimentaire essentiel pour la ville portuaire. Il représentait aussi une marchandise pour le commerce avec l'arrière-pays immédiat et les marchés urbains situés au-delà de la seule province de Castille. À partir du XIV siècle, la pêche prit la forme d'une activité économique à grande échelle, orientée vers la commercialisation des prises. Santander, Laredo et Castro Urdiales figurent déjà dans la liste des ports importants pour la pêche établie par les Cortes de Jerez de la Frontera en $1268^{34}$.

32. Arízaga Bolumburu, Beatriz, Solórzano Telechea, Jesús Angel, « Las actividades económicas de las villas medievales ", dans Historia de Cantabria, vol. I, Santander, El Diario Montañés, 2006, p. 209-216.

33. Cuñat CiscaR, Virginia, Documentación medieval de la villa de Laredo..., op. cit., p. 247.

34. Cortes de los antiguos reinos de León y Castilla, vol. I, Madrid, 1861, p. 74. 
Il existait deux saisons de pêche : l'une en hiver pour la daurade et l'autre au printemps pour la sardine. En outre, les innovations techniques permettaient aux pêcheurs d'aller de plus en plus loin, jusqu'en en Galice mais aussi en mer de Berbería ${ }^{35}$ et en Irlande. Par conséquent, ils restaient hors de leur ville pendant de longues campagnes qui duraient presque toute l'année, voire s'étendaient sur plusieurs années (deux, trois ou plus) ${ }^{36}$. Par exemple, les hommes de la confrérie de San Vicente de la Barquera s'embarquaient en mars pour aller pêcher en Irlande ou en Berbería et ne revenaient pas avant septembre. Thérèse, femme de Pierre Abaño, voisin de Saint-Vincent de la Barquera, déclare en 1500 que son mari ne devait pas payer l'impôt de la pêche vendue, puisqu'il avait été pendant trois ans hors de la ville : "Son mari était parti en mer pendant une année en naviguant, et deux années il est allé en Galice pour commercer, de sorte qu'il était resté hors de la ville pendant trois années, et pour cette raison il ne devait pas payer la alcabala, puisqu'il n'avait rien vendu dans la ville ${ }^{37}$."

Aux XIV et $\mathrm{XV}^{\mathrm{e}}$ siècles, les pêcheurs diversifiaient leurs activités essentiellement dans la sphère maritime. Ceci explique qu'il est parfois difficile d'établir une distinction nette entre pêcheurs, marins et marchands. Nous pouvons trouver des pêcheurs qui vendaient du poisson puis affrétaient leur bateau pour une transaction commerciale. Une autre activité, en plus de la pêche et de la vente du poisson, consistait à revendre sur place des cargaisons lors du retour au port d'attache. En 1486, Jean Fernand Galíndez, pêcheur de San Vicente de la Barquera, part avec son bateau "à la mer pour gagner la zone de pêche "; de retour de son voyage à Cantoviejo, il rapporte 120 douzaines de poissons qu'il vend 16000 maravédis ${ }^{38}$.

Ceux qui parmi les voisins exerçaient la profession de marchands n'appartenaient pas aux confréries de pêcheurs. Ainsi, Juan González del Corro, dit le chauve, voisin de San Vicente de la Barquera et membre de l'oligarchie urbaine, a soutenu un procès contre des pêcheurs de la confrérie de San Vicente qui avaient intentionnellement endommagé une pinasse appelée la Sainte-Marie dans la mer de Berbería. Les attaquants alléguaient que le procès devait être résolu par la confrérie de pêcheurs et que le maire de la ville n'avait pas les compétences requises dans les affaires en rapport avec la mer comme "il se disait dans les Lois d'Oléron " (Rôles d'Oléron), lois qui réglaient les affaires de la confrérie. De son côté, Juan González del Corro faisait valoir au contraire que la réglementation de la confrérie de pêcheurs ne le concernait pas parce qu'il était marchand ${ }^{39}$.

35. L'expression "mer de Berbería " désignait les eaux atlantiques au large du Maghreb.

36. LewIS, Archibald, The Sea and Medieval Civilization, Londres, Variorum Reprints, 1978 .

37. Solórzano Telechea, Jesús Angel, Vázquez, Roberto, Arízaga Bolumburu, Beatriz, San Vicente de la Barquera..., op. cit., doc. 49.

38. Ibidem, doc. 19.

39. Ibid., doc. 40. 
D'autre part, à partir du dernier quart du Xve siècle, diverses formes d'associations commerciales commencent à apparaître dans la documentation en relation avec la pêche à grande échelle. En général, les pêcheurs et les marins ne disposaient pas de moyens financiers suffisants pour armer des bateaux pendant de longues campagnes. Aussi, ils se sont souvent associés à des marchands pour financer les expéditions et répartir les risques que de telles entreprises faisaient courir. En 1498, Juan del Castillo, marchand de San Vicente de la Barquera, prête une quantité importante d'argent à Gonzalo Bravo, marin et pêcheur, pour aller en Galice ${ }^{40}$. Cette alliance était indispensable pour les pêcheurs, puisqu'ils trouvaient l'argent pour aller pêcher au loin. En 1486, le bachelier Alonso Ferrero, qui avait prêté 12000 maravedis à Jean Minguélez, pêcheur et voisin de San Vicente, pour équiper un baleinier pour une campagne à Cantoviejo, réclame son dû au retour du navire chargé de 120 douzaines de poissons pouvant valoir 16000 maravédis. Une fois l'emprunt déduit, les revenus furent répartis entre les deux associés ${ }^{41}$. De cette façon, la pêche qui, à l'origine, était plutôt une activité artisanale s'est peu à peu transformée en une activité industrielle ${ }^{42}$.

Les habitants des villes portuaires du Nord ont généralement pratiqué une double activité économique pendant tout le Moyen Âge, et même, dans quelques cas, une pluriactivité, soit plusieurs métiers ou activités professionnelles assurés de façon successive ou simultanée au cours de l'année par un seul individu.

Les activités économiques des élites urbaines étaient très différentes de celles du reste des autres groupes de citadins. Parmi le commun, il faut distinguer les omes ruanos qui avaient accès au marché de la terre de ceux qui en étaient exclus, les omes baxos. Les premiers exerçaient principalement des activités artisanales et mercantiles et occupaient une position économique qui leur permettait d'investir dans l'achat de terres ou de participer au financement d'entreprises de pêche.

L'essentiel de la population urbaine vivait de la pêche et appartenait au groupe des omes baxos. Certains pêcheurs développaient parfois des activités commerciales en relation avec des marchands (omes ruanos), mais très peu diversifiaient leurs activités en direction de la terre. Tournées vers la mer, leurs activités étaient étroitement encadrées et réglementées par les confréries de pêcheurs et de marins.

40. Ibid., doc. 21.

41. Ibid., doc. 12.

42. Arízaga Bolumburu, Beatriz, "La pesca en el País Vasco en la Edad Media ", Itsas Memoria, Revista de Estudios Marítimos Vascos, $\mathrm{n}^{\circ}$ 3, 2001, p. 13-28. FerReIRA PRIEgUe, Elisa, "O desenvolvemento da actividade pesqueira desde a Alta Idade Media ó século XVII ", dans Historia da pesca en Galicia, Santiago de Compostela, Universidad de Santiago de Compostela, 1998, p. 51-68. AzNAR VALLEJO, Eduardo, "Marinos y pescadores", Medievalismo, 2004, n 13-14, p. 229-240. 
En définitive, on peut affirmer - en relation avec l'hypothèse de départ de cet article - que seuls les omes ruanos et, à l'autre extrémité de l'échelle sociale, les journaliers ont pu développer une réelle pluriactivité, alors que la majorité des habitants n'a exercé que le métier de pêcheur.

\section{RÉSUMÉ}

Le présent article se propose d'analyser les différentes activités économiques des populations des villes portuaires de la Cantabrie au Moyen Âge dans le but d'étudier la manière dont elles assuraient leur subsistance et géraient les risques inhérents. Nous analyserons leurs activités principales, en particulier l'agriculture et l'exploitation des ressources offertes par la mer. Alors que la majorité des habitants a pratiqué une double activité tout au long du Moyen Âge seuls les groupes socio-économiques urbains de niveau intermédiaire ont développé une véritable pluriactivité.

\section{ABSTRACT}

This article attempts to define the various economic activities of the town-ports of Cantabria in order to determine the different ways to manage risk in the Middle Ages. Thus, author analyses the prevailing urban activities in these towns, with particular reference to the agricultural and maritime sector. Although most inhabitants practiced more than one economic activity throughout the Middle Ages, the author concludes that only the middle class developed what could be characterised as genuine pluriactivity. 